a Universidade Federal de São Carlos, Centro de Ciências e Tecnologias para a Sustentabilidade, Departamento de Ciências Ambientais, Campus Sorocaba, CEP 18052780 , Sorocaba-SP, Brasil.

' Universidade Federal de Lavras, Departamento de Automática, CEP 37200-900, Lavras-MG, Brasil

c Bioleucx, CEP 18150-000, Ibiúna-SP, Brasil

*E-mail: franciane@ ufscar.br

Recebido: 2 de Abril de 2021

Aceito: 17 de Maio de 2021

Publicado online: 28 de Julho de 2021

\section{Potencial Energético da Biomassa Residual do Processo de Extração do Óleo de Melaleuca}

\author{
Energy Potential of Residual Biomass from the Melaleuca Oil Extraction \\ Process
}

Sophya Lourenço Vasconcelos dos Santos, ${ }^{a}$ João Otávio Poletto Tomeleri, ${ }^{a}$ Fábio Domingues de Jesus, ${ }^{b}$ Rommel Sauerbronn da Cunha, c Fábio Minoru Yamaji,, Franciane Andrade de Pádua ${ }^{a, *(1)}$

Issues related to the study and characterization of alternative materials have been constantly addressed, with regard to the selection of strategic and most appropriate materials for energy applications. Thus, the energy use of processing waste is important for the circular economy. The Melaleuca alternifolia species is well-known for the production of oils with pharmaceutical and cosmetic properties, however its processing generates a large amount of residual biomass with little-known energy characteristics. The objective of this work was the characterization of the residual biomass from the extraction process of the essential oil of Melaleuca alternifolia Cheel with the purpose of application in bioenergy. The post-extraction residual material composed of leaves and stems, branches and bark (lenho) was separately characterized in terms of moisture, ash content, fixed carbon, volatile materials and superior calorific value (PCS). In addition, briquettes were manufactured with five different proportions of the residues (leaves and lenho) for the determination of their expansions and mechanical strengths. The briquettes of Melaleuca alternifolia Cheel presented mechanical resistance varying from $0,323 \mathrm{MPa}$ to $0,671 \mathrm{MPa}$. The leaf residues presented lower dry base moisture $(36.8 \%)$ and higher calorific value $(5.120 \mathrm{Kcal} / \mathrm{Kg})$ than the residual (lenho), however they presented a higher ash content $(4.11 \%), 61.6 \%$ higher than residual (lenho). The mechanical resistance of the briquette produced by $50 \%$ of the lenho and $50 \%$ of the leaf was superior to the other blends.

Keywords: Briquette; wastes; Melaleuca alternifolia; tea tree; bioenergy

\section{Introdução}

A Melaleuca alternifolia é uma espécie arbórea internacionalmente conhecida como tea tree, nativa da Austrália e Ilhas do Oceano Índico, pertencente à família Myrtaceae. A espécie possui interesse econômico por apresentar óleos voláteis, principalmente no seu tecido foliar. ${ }^{1}$ A espécie contém aproximadamente 100 componentes químicos oriundos de sua destilação a vapor. ${ }^{2}$ Além das propriedades farmacológicas, o óleo da melaleuca tem sido consumido em razão de suas propriedades antissépticas, para uso em cosméticos e produtos de higiene pessoal.

A $M$. alternifolia é uma árvore de rápido crescimento, podendo ser colhida aos seis meses de idade. ${ }^{3} \mathrm{O}$ maior produtor do óleo essencial da M. alternifolia é a Austrália, que detém o mercado mundial e as tecnologias de produção. ${ }^{4}$ No Brasil a produção ainda é pequena e a indústria que necessita desta matéria-prima ou de seus compostos isolados têm suprido a sua demanda por meio da importação. ${ }^{5}$ Os plantios da espécie no Brasil também são tímidos, sendo principalmente de pequenos produtores. As áreas plantadas no Brasil representam menos de 100 hectares, sendo 35 hectares no estado de São Paulo (Piedade, Ibiúna, São Pedro e Batatais), 12 hectares na Bahia (Ituaçú), 6 hectares no Mato Grosso (Chapada dos Guimarães) e 0,5 hectares no Mato Grosso do Sul (Maracaju).

O processo mais comum de extração do óleo essencial de melaleuca se dá por destilação por arraste a vapor a partir de toda a biomassa aérea (folhas, galhos e haste principal). O rendimento em óleo essencial de M. alternifolia é de aproximadamente 1 a $2 \%$ do peso fresco da planta, ${ }^{6}$ resultando em uma quantidade significativa de biomassa residual acumulada pós-extração. As pesquisas com a espécie são mais focadas nas características do óleo, poucos investigam os resíduos do processo. Dentre estas, se destacam as pesquisas que analisam as fibras do resíduo como reforço para biocompósitos. ${ }^{7,8}$ No Brasil, atualmente, o material residual é espalhado em campo visando a compostagem e ciclagem de nutrientes, no entanto, o processo é lento e não resolve o problema do acúmulo do resíduo. Uma das formas para resolver o problema é a sua utilização como biocombustível para a queima direta em caldeiras que fornecerão o 
vapor para o próprio processo de extração do óleo. Além disso, a transformação do resíduo in natura em produtos densificados, como os briquetes, trazem vantagens como contornar os problemas da baixa densidade energética do combustível, e melhorar suas características de transporte e alimentação em caldeiras.

Neste contexto, o objetivo deste trabalho foi investigar as características energéticas da biomassa residual da extração do óleo da espécie Melaleuca alternifolia Chell para o seu uso como combustível sólido.

\section{Material e Métodos}

O material residual do processo de extração do óleo de Melaleuca alternifolia, foi composto por folhas, ramos, hastes e fustes das plantas, provenientes de plantio pertencente ao Sítio das Melaleucas, localizado na cidade de Ibiúna, São Paulo, a 2339'23” de latitude sul, 47¹3'21" de longitude oeste, a de 996 metros de altitude. A biomassa residual do processo de extração do óleo das plantas por arraste a vapor foi coletada em destilaria localizada na mesma propriedade.

\subsection{Preparação e caracterização do material}

A biomassa residual do processo de extração do óleo da melaleuca foi separada em dois tipos de materiais: 1 . Folhas e 2. material lenhoso (hastes, ramos, fustes e casca). Cada tipo de resíduo foi triturado utilizando-se um moinho de rotor vertical com facas móveis e fixas tipo Willey MA-340. O material não passou pelo processo de separação granulométrica para que as análises pudessem ser condizentes com a realidade do produtor, diminuindo os custos do processo.

Para a análise química e determinação do teor de umidade, porções de cada tipo do material particulado foram utilizadas. Os materiais particulados foram levados à estufa de secagem com circulação de ar MA035/1 a $103^{\circ} \mathrm{C}$ $\pm 2^{\circ} \mathrm{C}$ até atingirem massa constante para cálculo do teor de umidade. A umidade do material foi aferida após três dias da colheita e extração do óleo essencial. O procedimento foi realizado em triplicata. A umidade em base úmida foi determinada de acordo com a Equação 1.

$$
\mathrm{Ubu}=(\mathrm{Pa} /(\mathrm{Pms}+\mathrm{Pa})) \times 100
$$

Em que:

Ubu = Umidade em base úmida, \%

$\mathrm{Pa}=$ Massa da água

Pms = Massa da matéria seca

A determinação do teor de materiais voláteis realizada nos resíduos previamente secos conforme prescrito na Norma ASTM E872-82, ${ }^{9}$ de acordo com a Equação 2.

$$
\mathrm{TMV}=(\mathrm{m} 2-\mathrm{m} 3) /(\mathrm{m} 2-\mathrm{m} 0) \times 100
$$

Em que:

TMV = Teor de materiais voláteis, \%

$\mathrm{m} 0=$ Massa do cadinho seco

$\mathrm{m} 2$ = Massa do cadinho + massa da biomassa

m3 = Massa do cadinho + massa da biomassa após mufla

O teor de cinzas foi determinado de acordo com a norma ASTM D1 102-84 ${ }^{10}$ e calculado de acordo com a Equação 3.

$$
\mathrm{TCz}=((\mathrm{Mac}-\mathrm{Mcad}) /(\mathrm{Mas})) \times 100
$$

Em que:

$\mathrm{TCz}=$ Teor de cinzas, $\%$

Mac $=$ Peso das cinzas + peso do cadinho

Mcad $=$ Peso do cadinho

Mas = Peso da amostra seca

O teor de carbono fixo foi calculado pela diferença entre os valores do teor de materiais voláteis e o de cinzas de acordo com a Equação 4.

$$
\mathrm{TCF}=100-(\mathrm{Tv}+\mathrm{Tc})
$$

Em que:

$\mathrm{TCF}=$ Teor de carbono fixo, $\%$

TMV $=$ Teor de material volátil, $\%$

$\mathrm{TCz}=$ Teor de cinzas, $\%$

O poder calorífico superior de cada tipo de biomassa residual foi obtido utilizando um calorímetro de marca IKA C200, com as amostras previamente secas em estufa, de acordo com a norma ASTM D2015-00. ${ }^{11}$ As análises ocorreram em triplicata.

\subsection{Produção e caracterização dos briquetes}

A briquetagem do material foi realizada por meio da aplicação, ao material poroso, a 12\% de umidade, de uma pressão de 15 toneladas por vinte segundos. $\mathrm{O}$ material então foi compactado e deu origem aos briquetes em formato cilíndricos, cujas medidas (altura e diâmetro) foram devidamente marcadas e cada briquete numerado, deixando-os expostos às condições do ambiente. Os tratamentos foram:

T1: $100 \%$ lenho

T2: $100 \%$ folha

T3: $50 \%$ folha $+50 \%$ lenho

T4: $75 \%$ folha $+25 \%$ lenho

T5: $25 \%$ folha $+75 \%$ lenho

No total foram confeccionados dez briquetes por tratamento totalizando 50 briquetes de 20 gramas. Os briquetes foram medidos imediatamente após a prensagem, após $30 \mathrm{~min}, 1 \mathrm{~h}, 4 \mathrm{~h}, 6 \mathrm{~h} 24 \mathrm{~h}$ e 48h, para análise da expansão longitudinal mediu-se a altura (direção de compactação) utilizando um paquímetro digital da marca INSIZE, modelo 1106-502, com capacidade de $150 \mathrm{~mm}$ e precisão de 
$0,01 \mathrm{~mm}$. Os briquetes foram mantidos em temperatura e umidade ambiente, sem qualquer controle climático.

O ensaio mecânico de tração por compressão diametral foi realizado em Máquina Universal de Ensaios EMIC DL30000N utilizando uma célula de carga com capacidade de $500 \mathrm{kgf}$ e velocidade de ensaio de $0,3 \mathrm{~mm} \cdot \mathrm{s}^{-1}$, conforme a norma ABNT NBR 7222, adaptada. ${ }^{12} \mathrm{O}$ ensaio foi realizado em dez briquetes por tratamento com o intuito de observar a resistência mecânica que o resíduo briquetado suporta e inferir acerca de seu comportamento no manuseio, transporte e queima.

\subsection{Análises estatísticas}

O delineamento experimental foi inteiramente casualizado, com 5 tratamentos. Respeitando-se as exigências estatísticas, os dados coletados foram submetidos ao Teste de Shapiro-Wilk, à análise de variância (ANOVA) e ao Teste de Tukey (com 5\% de significância). Todos os testes foram calculados com o software PAST. ${ }^{13}$

\section{Resultados e Discussão}

\subsection{Biomassa: umidade e análise imediata e poder calorífico superior}

A média das triplicatas da análise química imediata são apresentadas na Tabela 1 .

Os resíduos apresentaram umidade na base úmida de $36,86 \%$ e $42,32 \%$ para folha e lenho, respectivamente. Devido à natureza higroscópica da biomassa lenhosa, a umidade possui ampla variação no material e o teor de umidade ideal vai depender das dimensões da instalação que vai utilizá-la como fonte de combustível. Valores de umidade base úmida de 49,04\% foram verificados para cavacos de eucalipto recém colhido ${ }^{14} \mathrm{e}$ de $54 \%$ em cavacos de madeira de pinus. ${ }^{15} \mathrm{Em}$ comparação ao bagaço de cana a literatura demonstra valores de umidade de $50 \%{ }^{16} \mathrm{e}$ de 28 a $31 \%{ }^{17}$. Altos teores de umidade implicam em redução do poder calorífico e maiores custos de manejo e transporte da biomassa.

O teor de materiais voláteis dos resíduos pós-extração do óleo, foram de 76,09\% e 79,03\% para folha e lenho, respectivamente. Em comparação à biomassa lenhosa de eucalipto, são relatados valores de $82,99 \%{ }^{18}$ e $84,2 \pm$ $0,23 \% .{ }^{19} \mathrm{Em}$ comparação ao bagaço de cana, são relatados valores de 81,7 a 69,4\% considerando a biomassa no início e após 10 dias de secagem, respectivamente. ${ }^{17}$

O processo prévio de extração de óleo a que a biomassa de $M$. alternifolia foi submetida pode ter reduzido o teor de voláteis no material residual. O teor de carbono fixo da biomassa residual de M. alternifolia foi de $19,79 \%$ e $19,36 \%$ nas folhas e lenho, respectivamente. Esses valores foram superiores quando comparados a biomassa de cavacos de eucalipto de $14,8 \pm 0,23 \%)^{19}$ e $15,63 \% .{ }^{18}$ Combustíveis com menores teores de materiais voláteis e altos valores de carbono fixo tendem a ter maior tempo de queima em fornos, uma vez que o processo ocorre de forma mais lenta. ${ }^{20}$

$\mathrm{O}$ teor de cinzas da biomassa residual de $M$. alternifolia foi de $4,12 \%$ e $1,60 \%$ nas folhas e material lenhoso, respectivamente. Valores de 1,38\% em cavacos de eucalipto com casca são relatados. ${ }^{18} \mathrm{Em}$ comparação ao bagaço residual de cana, foram relatados valores de $5,57 \%$ de cinzas ${ }^{21}$ e valores de 3,30\% na biomassa recém processada e 7,21\% após 15 dias de secagem. ${ }^{17}$ As cinzas são os materiais inorgânicos residuais após a combustão completa do material combustível. Menores valores de cinzas implicam em maior poder calorífico e menores problemas de corrosão nos sistemas de transformação. Os valores para o teor de cinzas de cavacos de eucaliptos podem variar de 2 a $20 \%$, dependendo da situação da biomassa. ${ }^{22}$

O poder calorífico da biomassa residual da extração do óleo a M. alternifolia foi de $4.838 \mathrm{kcal} / \mathrm{kg}$ para o lenho e $5.121 \mathrm{kcal} / \mathrm{kg}$ para a folha, respectivamente. Os resultados encontrados são superiores aos $3.936 \mathrm{kcal} / \mathrm{kg}$ descritos para o bagaço de cana ${ }^{21}$ e superiores aos $4033 \mathrm{kcal} / \mathrm{kg}$ para cavacos de eucalipto. ${ }^{19}$

\subsection{Briquetes: comportamento higroscópico, densidade e resistência mecânica}

A Figura 1 A e B ilustra o comportamento higroscópico dos briquetes considerando a seção diametral.

A maior ou menor expansão dos briquetes pode ser explicada pela adesão das partículas, influenciando na menor resistência mecânica dos briquetes com os maiores particulados. Pode-se perceber que o tratamento $1(100 \%$ lenho) foi o que apresentou os piores resultados expansão em detrimento do T2 (100\% folhas) o qual apresentou as menores expansões. Os resultados apresentados na Figura 1, demonstram que a adição de folhas ao conteúdo lenhoso melhora a adesão entre as partículas, reduz a expansão diametral dos briquetes e, consequentemente, melhora as características de transporte e manuseio do material.

\subsection{Resistência mecânica dos briquetes}

A Tabela 2. apresenta os resultados para a resistência mecânica dos briquetes fabricados com diferentes porcentagens de folhas e lenho, compreendendo os cinco tratamentos.

Tabela 1. Valores médios de umidade e análise imediata da biomassa residual do processo de extração do óleo de Melaleuca alternifolia

\begin{tabular}{lccccc}
\hline Biomassa & Ubu (\%) & TMV (\%) & TCz $(\%)$ & TCF $(\%)$ & PCS $($ Kcal/Kg) \\
\hline Folhas & 36,85 & 76,09 & 4,12 & 19,78 & 5.121 \\
Lenho & 42,32 & 79,03 & 1,60 & 19,36 & 4.838
\end{tabular}

Ubu: umidade inicial base úmida; TCz: teor de cinzas; TCF: teor de carbono fixo; TMV: teor de materiais voláteis; PCS: poder calorífico superior 


\section{Expansão Diametral dos Tratamentos}

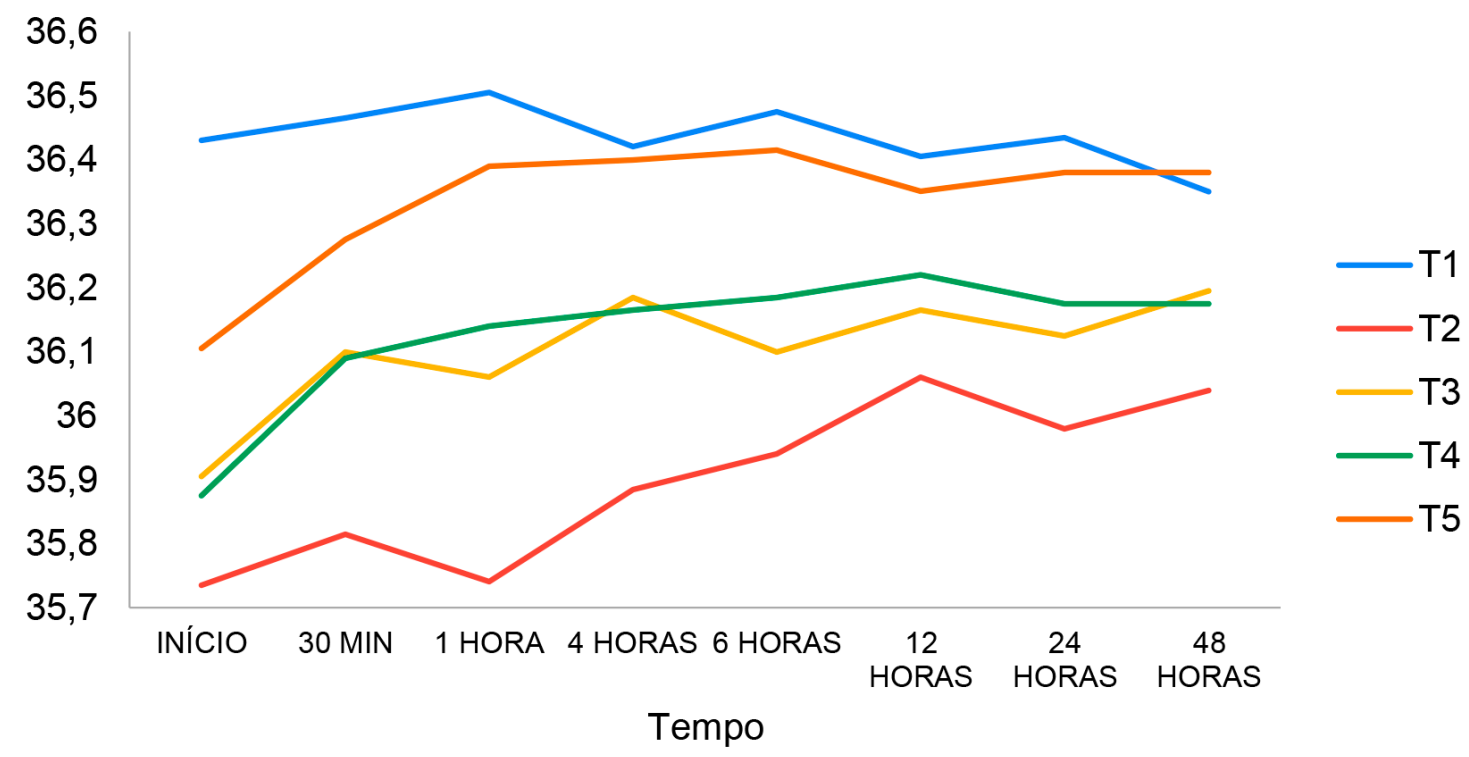

B

\section{Expansão Vertical dos Tratamentos}

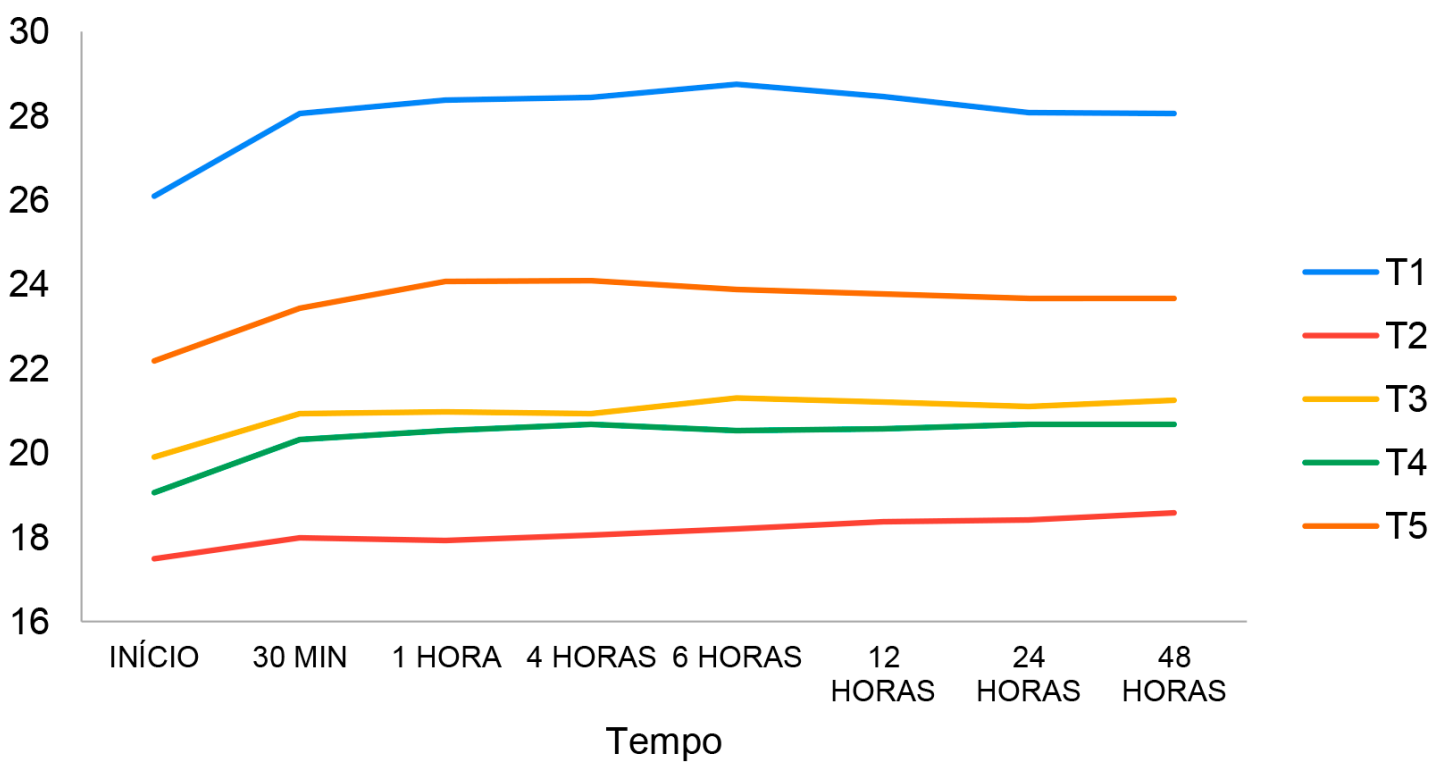

Figura 1. Expansão diametral (A) e expansão longitudinal dos briquetes (B)

Tabela 2. Resistência mecânica por compressão diametral de briquetes fabricados a partir de diferentes porcentagens de folhas e material lenhoso de biomassa residual da extração do óleo de Melaleuca alternifolia

\begin{tabular}{lccc}
\hline Tratamento & Força Máxima $(\mathbf{k g f})$ & Resistência Máxima $(\mathbf{M P a})$ & Densidade $\left(\mathbf{g} / \mathbf{c m}^{\mathbf{3}}\right)$ \\
\hline T1 & $431 \mathrm{c}$ & $0,323 \mathrm{c}$ & $0,73 \mathrm{~b}$ \\
T2 & $677 \mathrm{~b}$ & $0,509 \mathrm{~b}$ & $1,13 \mathrm{a}$ \\
T3 & $892 \mathrm{a}$ & $0,671 \mathrm{a}$ & $0,98 \mathrm{~b}$ \\
T4 & $628 \mathrm{~b}$ & $0,546 \mathrm{~b}$ & $1,02 \mathrm{a}$ \\
T5 & $734 \mathrm{~b}$ & $0,552 \mathrm{~b}$ & $0,87 \mathrm{~b}$ \\
\hline
\end{tabular}

Médias seguidas pela mesma letra na coluna não diferem entre si a 5\% de significância pelo teste de Tukey 
Briquetes produzidos a partir de serragem de eucalipto e pinus, bagaço e palha de cana de açúcar apresentaram resistência mecânica variando de $1,215 \mathrm{MPa}$ (serragem de eucalipto) a 0,270 MPa (bagaço de cana de açúcar). ${ }^{23}$ Sendo assim, os resultados encontrados para o presente estudo encontram-se em uma média intermediária variando de 0,323 MPa a 0,671 MPa. Os briquetes produzidos apenas com biomassa lenhosa (hastes, ramos e cascas) tiveram menores densidades e resistências mecânicas. Indicando que a biomassa foliar foi responsável pela melhoria das propriedades mecânicas do combustível.

Considerando as variáveis analisadas, o tratamento composto por $50 \%$ de material lenhoso e $50 \%$ de folhas apresentou a maior resistência mecânica, sendo indicado como a mistura de biomassas que produz briquetes com melhores propriedades para o manuseio e transporte.

\section{Conclusões}

A biomassa residual da extração do óleo de Melaleuca alternifolia apresenta potencial energético, com um poder calorífico comparado ao de biomassas de uso consagrado para tal fim. Isso é particularmente importante ao se considerar a biomassa residual gerando energia de qualidade para o próprio processo de extração do óleo, envolvendo os conceitos de economia circular. Visando ainda agregar valor ao resíduo, a briquetagem se mostrou uma alternativa para o uso energético da biomassa, considerando a compactação de $50 \%$ de folhas e $50 \%$ de material lenhoso (hastes, cascas e galhos), ponderando a análise química, poder calorífico, expansão, resistência mecânica dos briquetes e, consequentemente, suas características energéticas e de manejo e transporte.

\section{Agradecimentos}

Os autores agradecem ao CNPq pela concessão de bolsa de estudo, ao Bioleucx, Sítio das Melaleucas e ao Grupo de Pesquisa em Biomassa e Bioenergia da UFSCar.

\section{Referências Bibliográficas}

1. Martins, J. A. S.; Sagata, E.; Santos, V. A.; Juliatti, F. C.; Avaliação do efeito do óleo de Melaleuca alternifolia sobre o crescimento micelial in vitro de fungos fitopatogênicos. Bioscience Journal 2011, 27, 49. [Link]

2. Hammer, K. A.; Carson, C. F.; Riley, T. V.; Antifungal effects of Melaleuca alternifolia (tea tree) oil and its components on Candida albicans, Candida glabrata and Saccharomyces cerevisiae. Journal of Antimicrobial Chemotherapy 2004, 53, 1081. [CrossRef]

3. Huynh, Q.; Phan, T.D.; Thieu, V. Q. Q.; Tran, S. T.; Do, S. H. Extraction and refining of essential oil from Australian tea tree, Melaleuca alterfornia, and the antimicrobial activity in cosmetic products. Journal of Physics: Conference Series 2012, 352, 1. [CrossRef]
4. Castro, C.; Silva, M. L.; Pinheiro, A. L.; Jacovine, L. A. G.; Análise econômica do cultivo e extração do óleo essencial de Melaleuca alternifólia Cheel. Revista Árvore 2005, 29, 241. [CrossRef]

5. Castelo, A. V. M.; Afonso, S. R.; Melo, R. R.; Del Menezzi, C. H. S.; Camillo, J.; Vieira, R. F.; Rendimento e composição química do óleo essencial de Melaleuca alternifólia Chell, na região do Distrito Federal. Agrária - Revista Brasileira de Ciências Agrárias 2013, 8, 143. [CrossRef]

6. Carson, C. F.; Riley, T. V.; Antimicrobial activity of the essential oil of Melaleuca alternifolia. Letters in Applied Microbiology 1993, 16, 49. [CrossRef]

7. Rodney J.; Sahari, J.; Kamal, M. S. M.; Sapuan, S. M.; Thermochemical and mechanical properties of tea tree (Melaleuca alternifolia) fibre reinforced tapioca starch composites. e-Polymers 2015, 15, 401. [CrossRef]

8. Rodney J.; Sahari J.; Kamal, M. S. M.; Physicochemical and Mechanical Properties of Different Morphological Parts of the Tea Tree (Melaleuca alternifolia) Fibres. Fibres and Textiles in Eastern Europe 2015, 114, 31. [Link]

9. Sítio ASTM Internacional. ASTM E872-82: Standard test method for volatile matter in the Analysis of particulate wood fuels. Disponível em: <https://www.astm.org/Standards/E872.htm>. Acesso em: 11 junho 2021.

10. Sítio ASTM Internacional. ASTM D1102-84: Standard test method for ash in wood. Disponível em: $<$ https://www.astm.org/ Standards/D1102.htm>. Acesso em: 11 junho 2021.

11. Sítio ASTM Internacional. ASTM, D2015-00 - Standard Test Method for Gross Calorific Value of Coal and Coke by the Adiabatic Bomb Calorimeter (Withdrawn 2000). Disponível em: $<$ https://www.astm.org/Standards/D2015.htm>. Acesso em: 11 junho 2021.

12. Sítio ABNT Catálogo, ABNT NBR 7222 - Determinação da resistência à tração por compressão diametral de corpos de prova cilíndricos. Disponível em: <https://www.abntcatalogo.com.br/ norma.aspx?ID=87151>. Acesso em: 11 junho 2021.

13. Valentim, L. B.; Tomeleri, J. O. P.; Thiersch, C. R.; Thiersch, M. F. B. M.; Alesi, L. S.; Varanda, L. D.; Almeida, R. E. P.; Yamaji, F. M.; Pádua, F. A.; Mapping Three-dimensional Moisture Content of Wood Chip Piles for Energy Production. Floresta e Ambiente 2019, 26, 1. [CrossRef]

14. Furtado, T. S.; Ferreira, J. C.; Brand, M. A.; Muñiz, G. I. B.; Quirino, W. F; Mapeamento da frequência de uso e características da biomassa florestal utilizada para geração de energia em Lages, SC. Ciência Florestal 2012, 22, 795. [CrossRef]

15. Panchal R. J.; Shinde, S. M.; Panchal, S. J.; Effect of Bagasse Moisture on Boiler Performance. International Research Journal of Multidisciplinary Studies 2016, 2, 1. [Link]

16. Omoniyi, T.E., Olorunnisola A. O.; Experimental Characterisation of Bagasse Biomass Material for Energy Production. International Journal of Engineering and Technology 2014, 4, 582. [Link]

17. Valentim, L. B.; Dissertação de Mestrado, Universidade Federal de São Carlos, 2016. [Link]

18. Ferreira, I. T. F., Schirmer, W. N., Machado, G. O., Gueri, M. V. D.; Estimativa do potencial energético de resíduos celulósicos de fabricação de papel através de análise imediata. Revista Brasileira de Energias Renováveis 2014, 3, 284. [CrossRef] 
19. Brand, M. A.; Energia de biomassa florestal, 2010. Rio de Janeiro: Interciência, 2010.[Link]

20. Fernandez, B. O., da Róz, A. L., Gonçalves, B. F., Nakashima, G. T., Yamaji, F. M.; Qualidade de Briquetes de Cana-de-Açúcar Produzidos com Aglutinante Amido de Milho. Revista Virtual de Química 2018, 10, 142. [CrossRef]
21. Alakangas, E.; Properties of Wood fuels used in Finland. Technical Research of Finland, Project report PRO2/P2030/05. Disponível em: <https://pt.scribd.com/document/335110364/ bio-south-wood-fuel-properties-pdf $>$. Acesso em 11 junho 2021.

22. Silva, D. A. da; Yamaji, F. M.; Barros, J. L.; Róz, A. L.; Nakashima, G. T.; Caracterização de biomassa para a briquetagem. Floresta 2015, 45, 713. [CrossRef] 